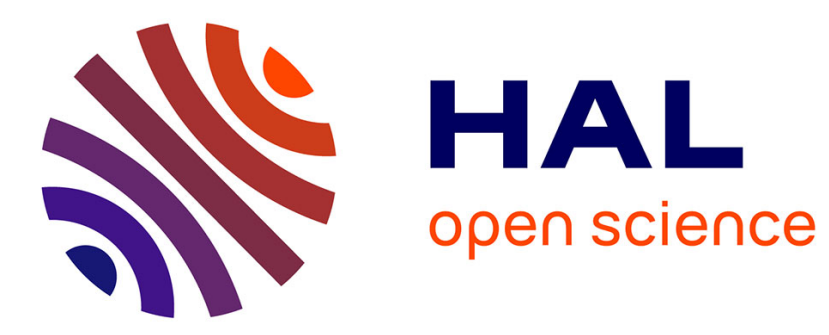

\title{
A theory of spatial general equilibrium in a fuzzy economy
}

Claude Ponsard

\section{To cite this version:}

Claude Ponsard. A theory of spatial general equilibrium in a fuzzy economy. [Research Report] Institut de mathématiques économiques ( IME). 1984, 25 p., bibliographie. hal-01542396

\section{HAL Id: hal-01542396 https://hal.science/hal-01542396}

Submitted on 19 Jun 2017

HAL is a multi-disciplinary open access archive for the deposit and dissemination of scientific research documents, whether they are published or not. The documents may come from teaching and research institutions in France or abroad, or from public or private research centers.
L'archive ouverte pluridisciplinaire HAL, est destinée au dépôt et à la diffusion de documents scientifiques de niveau recherche, publiés ou non, émanant des établissements d'enseignement et de recherche français ou étrangers, des laboratoires publics ou privés. 


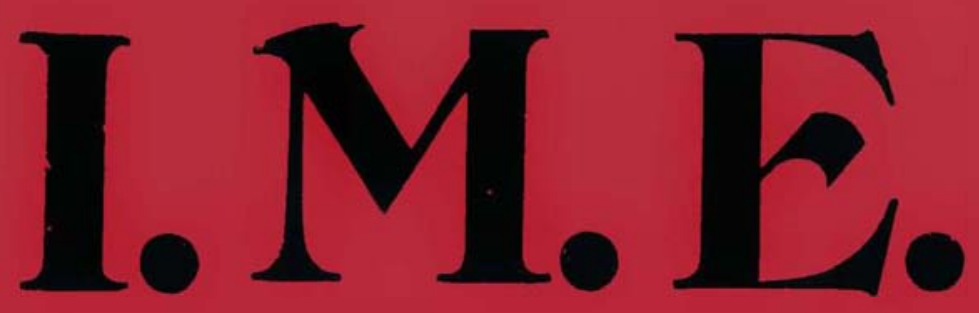

EQUIPE DE RECHERCHE ASSOCIEE AU C.N.R.S.

DOCUMENT DE TRAVAIL

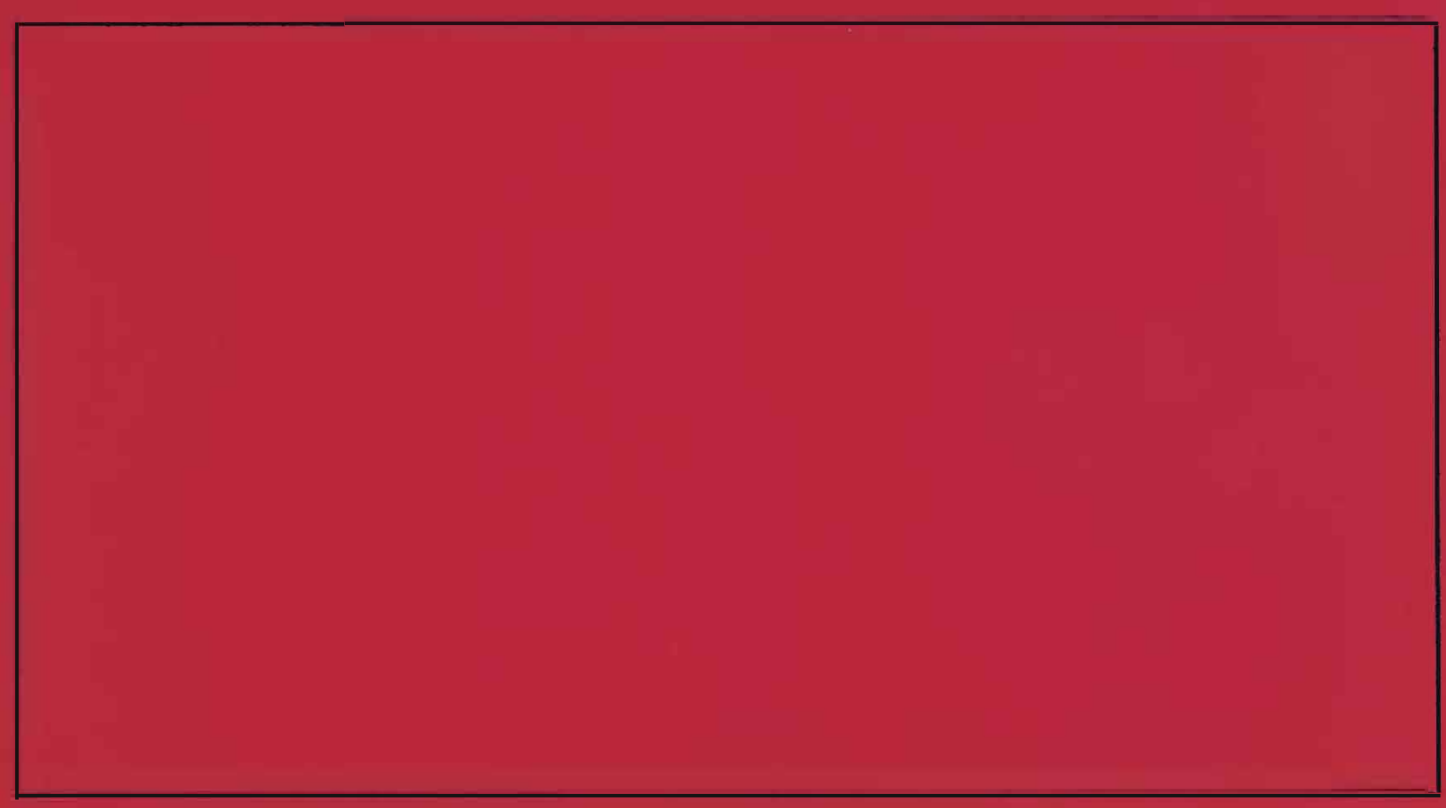

INSTITUT DE MATHEMATIQUES ECONOMIQUES

UNIVERSITE DE DIJON

FACULTE DE SCIENCE ECONOMIQUE ET DE GESTION

4. BOULEVARD GABRIEL -21000 DIJON 
No 65

A THEORY OF SPATIAL GENERAL EQUILIBRIUM

IN A FUZZY ECONOMY

Claude PONSARD

April 1984

This paper is to be presented at the Second International Congress of Arts and Sciences, Regional Science Sessions, to be held June 3-16, 1984, at the Erasmus University, Rotterdam, The Netherlands. 


\title{
A THEORY OF SPAIIIAL GENERAL EQUIL IBRIUM IN A FUZZY ECONOMY
}

\author{
Claude PONSARD \\ Professor at the University of Dijon \\ Director of the Institute of Economic Mathematics \\ (National Center for Scientific Research) \\ 4, boulevard Gabriel - 21000 DIJON (France)
}

\begin{abstract}
.
Let an economic space be characterized by the existence of a given distribution of locations, i.e. consumers' residential locations and producers' plants. It is equipped with a system of prices. The economy is fuzzy because the economic behaviors of agents are imprecise.

In this context, spatial partial equilibria theories are applications of a fuzzy economic calculation model. The aim of the present paper is to study the conditions which must be fulfilled in order that the compatibility of consumers' equilibria and producers' equilibria be verified.

Mathematical tools are Butnariu's theorems which extend the Brouwer's and Kakutani's theorems to the cases of fuzzy functions and fuzzy point-to-set mappings.

Economic results are the extension of the Walras Law to a fuzzy economic space and the formulation of a theorem which states the conditions for the existence of a spatial general equilibrium in a fuzzy economy. This theorem is a generalization of a classical Debreu's result.
\end{abstract}

Keywords : Economic Space, General Equilibrium, Fuzzy Sets, Mathematical Economics.

\section{Introduction.}

1.1. A fuzzy economy is such that : (1) economic spaces where men live (regions, market areas, urban influence zones, etc.) are imprecise ; they 
possess "more or less" constituent characteristics and they are not partitioned into very homogeneous and well delineated sub-spaces ; (2) economic agents pursue vague and sometimes incompatible objectives ; the constraints which limit their means are elastic, either because they are imperfectly appraised, or because they are objectively fuzzy.

The matter of the fuzzy economic spaces analysis is to state a formalized theory which retains in full the nuances and gradations of the real world and the lights and shades of the spatial human behaviors (Ponsard, 1980, 1983).

1.2. With respect to spatial behaviors, they must be analysed as fuzzy economic calculations, i.e. as optimization programmings of fuzzy objective-functions with elastic constraints. Then, consumers and producers spatial partial equilibria theory is a particular specification of the fuzzy economic calculation general model. Consumers optimal decisions are their demands and producers optimal decisions are their supplies.

1.3. The aim of this paper is to study the compatibility of fuzzy spatial partial equilibria, i.e. to state a theory of spatial general equilibrium in a fuzzy economy. More precisely, this paper is limited to the analysis of the existence conditions of such an equilibrium.

The first part is devoted to the description of the analysis framework ; the matter of the second part is to state a theorem of equilibrium existence.

1.4. Remark : In order to avoid any ambiguity in the notation of mathematical symbols, ordinary concepts (non fuzzy) are underlined, whereas fuzzy concepts are not. For instance, $A \subset \underline{E}$ is read : A is a fuzzy subset of the ordinary reference set $\underline{E}$. This convention is only applied to sets and not to their elements. No ambiguity is possible. 


\section{The analysis framework.}

2.1. The characteristics of the economic space.

Let an economic space characterized by $n$ goods $i(i=1, \ldots, n), m$ consumers $j(j=1, \ldots, m)$ and $r$ producers $k(k=1, \ldots, r)$. The set of agents is finite, but large enough in order that the economy has a structure of competition.

The locations of economic agents are given and their distribution is indifferent. We note without any ambiguity the consumers residences with the help of the same indexes $j$, one and only one individual residence corresponding to each consumer. Thus, different values of these indexes are allocated to distinct residences which are located in a given place or in several places. In the same manner, producers plants are noted with the help of indexes $k$.

Although the following model is inspired by the Debreu's theory (1959), the definition of an economic good which is chosen is not that of a merchandise in the meaning stated by this author. It is well known that such a merchandise is described as a product or a service which is characterized by various properties, especially the date to which it will be available and the place where it will be accessible. Then, the value of a merchandise in the future is equalized with the value of the same merchandise at present through a discount rate. In the same manner, the value of a distant merchandise is supposed to be equalized with the value of the same merchandise which is available in a given place through an exchange rate.

But, if it is possible to state the present value of a merchandise available in the future with the help of a discount rate, the same thing is not accurate to state the local value of a distant merchandise with the help of an exchange rate. Indeed, the actualisation calculation is based upon the axiom of the depreciation of the future which lays the foundation of the exchange rate. For lack of a similar axiom in spatial analysis, Debreu's exchange rate has no theoretical foundation. Thenceforth, this definition of a merchandise does not allow to explicit the impact of space in the economic equilibria. Therefore the usual definition of an economic good must be kept. We call "located goods" the nonspatial goods equipped with the coordinates of their supply or demand places in economic space. 
Then, the economic space is characterized by a price system which allows to state the nominal value of physical products and services at a given instant. The delivered price of a $i^{\text {th }}$ good unit which is demanded by the $j^{\text {th }}$ consumer (located at the place $j$ ) to the $k^{\text {th }}$ producer (located at the place $k$ ) is denoted by $p_{i j k}$. It is equal to the sum of the factoryprice and the transportation price from $k$ to $j$ :

$$
p_{i j k}=p_{i k}+p_{i \cdot k j}
$$

where $p_{i k}$ is the factory-price of the good $i$ at place $k$ and $p_{i ' k j}$ the transportation price of the good $i$ from $k$ to $j$, with $i$ ' which designates the transportation service of the good $i$.

A price-vector, denoted by $p, p=\left[p_{i j k}\right]$, describes a spatial price system. The set of prices is denoted by $\underline{P}$. Thus $: p \in \underline{P}$.

We put the assumption that one price at least is not null. Subsequently, this condition will be necessary to verify a generalized Walras Law.

\subsection{The description of the fuzzy economy.}

2.2.1. Imprecise preferences, fuzzy utility, sets of located possible and efficient consumptions.

Let $\underline{x}_{j}$ be the set of the located possible consumptions of the $j$ th consumer. The elements $x_{j}$ of $\underline{x}_{j}$ are vectors of $\mathbb{R}^{n m r}$ whose compoundings $x_{i j k}$ state the quantities of the good $i$ which are demanded or supplied by the $j^{\text {th }}$ consumer to the $k^{\text {th }}$ producer. They are non-negative or non-positive according as they are demanded or supplied respectively. $\mathbb{R}^{1}$

To simplify, we put $: n m r=1$. The elements $x_{j}$ of $\underline{x}_{j}$ are vectors of

The $j^{\text {th }}$ consumer has an imprecise preference-indifference system described by a fuzzy total preorder (Ponsard, 1981 a). Under some conditions which assure the existence of a topological totally preordered space, a fuzzy continuous utility function, denoted by $\mu_{u_{j}}$, is stated. It is such
that :

$$
\mu_{U_{j}}: \underline{x}_{j} \longmapsto[0,1]
$$




$$
\forall x_{j} \in \underline{x}_{j}, x_{j} \longmapsto \mu_{u_{j}}\left(x_{j}\right) \in[0,1]
$$

Thus we define a fuzzy subset of $\underline{x}_{j}$, denoted by $U_{j}$, such that :

$$
U_{j}=\left\{x_{j}, \mu_{U_{j}} ; \forall x_{j} \in \underline{x}_{j}: \mu_{u_{j}}\left(x_{j}\right) \in[0,1]\right\}
$$

which describes a fuzzy objective in the $j^{\text {th }}$ consumer's economic calculation.

The $j^{\text {th }}$ consumer disposes of a given budget, represented by the real $w_{j} \in \mathbb{R}$. For a set $\underline{x}_{j}$, a spatial price system $p$ and a wealth $w_{j}$, the set of budget is defined by :

$$
\underline{B}_{j}=\left\{x_{j} ; \forall x_{j} \in \underline{x}_{j}: p \cdot x_{j} \leqslant w_{j}\right\}
$$

where p. $x_{j} \leqslant w_{j}$ designates the budget constraint.

But, except for some particular cases, it is elastic, so that the located efficient consumptions set (for a given constraint $\underline{B}_{j}$ ) is no more reduced to the classical frontier of the consumption technical optima. It is a fuzzy subset of $\underline{x}_{j}$, since each element $x_{j}$ belongs "more or less" to $\underline{x}_{j}$. Thus, we construct a mapping, denoted by $\mu_{c_{j}}$, such that :

$$
\begin{aligned}
& \mu_{c_{j}}: \underline{x}_{j} \longmapsto[0,1] \\
& \forall x_{j} \in \underline{x}_{j}, x_{j} \longmapsto \mu_{c_{j}}\left(x_{j}\right) \in[0,1] \\
& \text { and } \mu_{C_{j}}\left(x_{j}\right)=1 \text { if } p \cdot x_{j}=w_{j} \\
& =0 \text { if } p \cdot x_{j}>w_{j} \\
& \left.{ }^{\mu_{C_{j}}}\left(x_{j}\right) \in\right] 0,1\left[\text { if } p \cdot x_{j}<w_{j}\right.
\end{aligned}
$$

The mapping $\mu_{C_{j}}$ is dependent on $w_{j}$. It is monotonous and decreasing 
for increasing values of $w_{j}:\left(w^{\prime}{ }_{j}>w_{j}\right) \Rightarrow\left[\left(w_{j}^{\prime}-p \cdot x_{j}\right)>\left(w_{j}-p \cdot x_{j}\right)\right]$ $\Rightarrow \mu^{\prime} C_{j}\left(x_{j}\right) \leqslant \mu_{j}\left(x_{j}\right)$

Thus, we define a fuzzy subset of $\underline{x}_{j}$, denoted by $c_{j}$, such that : $c_{j}=\left\{x_{j}, \mu_{c_{j}} ; \forall x_{j} \in \underline{x}_{j}: \mu_{c_{j}}\left(x_{j}\right) \in[0,1]\right\}$

which describes a fuzzy constraint in the $j$ th consumer's economic calculation.

2.2.2. Producers fuzzy objectives, sets of possible and efficient productions.

Let $\underline{Y}_{k}$ be the set of the possible productions of the $k$ th producer. The elements $y_{k}$ of $\underline{Y}_{k}$ are vectors of $\mathbb{R}^{l}$ whose compoundings $y_{i j k}$ state the quantities of the good $i$ which are supplied or demanded by the $k^{\text {th }}$ producer to the $\mathrm{jth}$ consumer. They are non-negative or non-positive according as they are supplied or demanded respectively.

Transportations are production activities just like all the others.

For a production $y_{k}$ in $\underline{Y}_{k}$ and a spatial price system $p$, the producer's profit, denoted by $\underline{G}_{k}$, is by definition :

$$
\underline{G}_{k}=p \cdot y_{k}
$$

The objective of the $k^{\text {th }}$ producer is the maximization of the fuzzy utility associated with the profit (Ponsard, 1982 a).

We construct a mapping, denoted by $\mu_{G}$, which is the profit fuzzy utility mapping. It is such that :

$$
\mu_{G}: \underline{Y}_{k} \longmapsto[0,1]
$$

$\forall y_{k} \in \underline{Y}_{k}, y_{k} \longmapsto \mu_{G_{k}}\left(y_{k}\right) \in[0,1]$ 
and $\mu_{G_{k}}\left(y_{k}\right)=0$ ir the inaction assumption

$$
\left.\left.\mu_{G_{k}}\left(y_{k}\right) \in\right] 0,1\right] \text { in all the other cases. }
$$

We find again, as a particular case, the classical assumption if the profit utility is maximal $\left(\mu_{G_{k}}\left(y_{k}\right)=1\right)$ for the maximum amount of profit.

Thus, we define a fuzzy subset of $G_{k}$, denoted by $G_{k}$, which describes the fuzzy objective of the $k$ th producer. It is such that :

$$
G_{k}=\left\{y_{k}, \mu_{G_{k}} ; \forall y_{k} \in \underline{Y}_{k}: \mu_{G_{k}}\left(y_{k}\right) \in[0,1]\right\} .
$$

Now, instead of partioning the set $\underline{Y}_{k}$ into two classes, for a given technological constraint, that of efficient productions and its complemental class in $\underline{Y}_{k}$, we admit that all the elements of $\underline{Y}_{k}$ are "more or less" efficient productions.

Thus we construct a membership function of the elements of $\underline{Y}_{k}$, denoted by $\mu_{H_{k}}$, such that :

$$
\mu_{H_{k}}: \underline{Y}_{k} \longmapsto[0,1]
$$

$\forall y_{k} \in \underline{y}_{k}, y_{k} \longmapsto \mu_{H_{k}}\left(y_{k}\right) \in[0,1]$

with $\mu_{H_{k}}\left(y_{k}\right)=0$ in the assumption of the production of wastes

$\mu_{H_{k}}\left(y_{k}\right)=1$ in the classical case of maximal efficiency

$\left.\mu_{H_{k}}\left(y_{k}\right) \in\right] 0,1[$ in all the other cases.

We define a fuzzy subset of $\underline{Y}_{k}$, denoted by $H_{k}$, such that :

$$
H_{k}=\left\{y_{k}, \mu_{H_{k}} ; \forall y_{k} \in \underline{Y}_{k}: \mu_{H_{k}}\left(y_{k}\right) \in[0,1]\right\}
$$


which describes a fuzzy constraint in the $k^{\text {th }}$ producer's economic calculation.

\subsubsection{Allocation of wealths and incomes.}

The initial allocation of the stocks of goods $i$ is given. Let $w_{j}$ be the vector of the $j$ th consumer resources and $w=\sum_{j=1}^{m} w_{j}$ the initial wealth in the economy.

The distribution of profit between the consumers is defined by the parts, denoted $\lambda_{k j}$, of the net revenues of firms which are perceived by the consumers. These coefficients are assumed to be non-negative and their sum equal to one for the $r$ firms in the economy.

With these hypotheses, each consumer has three different origins for his incomes : incomes proceeding from its profit parts, from the sale of its initial dotations and from the sale of the services which are included in its consumption set.

We state the hypothesis that each agent can supply a quantity of each good in order to avoid discontinuity solutions :

$$
w_{j} \in \mathbb{R}^{n} \text { and } \exists x_{j} \in \underline{x}_{j} / x_{j}<w_{j} \cdot
$$

2.3. Definition of the spatial general equilibrium in a fuzzy economy.

\subsubsection{Fuzzy economic calculation.}

Let $\underline{E}, \underline{E}=\{x\}$, be the set of a priori possible alternatives. A fuzzy decision, denoted by $D$, in $\underline{E}$ is by definition the intersection of the fuzzy subset $F, F \subset \underline{E}$, describing the aimed objective (the set of the "more or less" attained objective values) and of the fuzzy subset $C, C \subset \underline{E}$, describing the constraint (the set of the "more or less" satisfied constraint values):

$$
D=F \cap C
$$

We have a membership function, denoted by $\mu_{0}$, such that :

$$
\mu_{D}: \underline{E} \longrightarrow[0,1]
$$


$\forall x \in \underline{E}, x \longmapsto \mu_{D}(x)=\mu_{F}(x) \wedge \mu_{c}(x)$

with the following conditions :

$\mu_{D}(x) \simeq 1$ iff $x$ is good for $F$ and $C$

$\mu_{D}(x) \simeq 0$ iff $x$ is bad for $F$ or $C$

An optimal decision is such that :

$$
\sup _{x \in \underline{E}} \mu_{D}(x)=\sup _{x \in \underline{E}}\left[\mu_{F}(x) \wedge \mu_{c}(x)\right]
$$

This formulation sends for a very important remark in the framework of spatial partial equilibria theories : objective and constraint have the same part in the decision-making process. Indeed, their relationships are symmetric since two fuzzy subsets of the same reference set are concerned and the operation $n$ is commutative.

Formally, a fuzzy economic calculation has a structure which is similar with the structure of a fuzzy mathematical programming. Tanaka, Okuda and Asai (1974) prove that the solution for the problem of finding the best possible decision is to settle an element $x$ of $\underline{E}$ such that :

$$
\begin{aligned}
& \sup _{x \in \underline{E}} \mu_{D}(x)=\sup _{x \in \underline{A}} \mu_{\boldsymbol{F}}(x) \text {, with } \underline{A} \subset \underline{E} \text { and } \\
& \underline{A}=\left\{x ; x \in \underline{E}: \mu_{C}(x) \geqslant \mu_{F}(x)\right\}
\end{aligned}
$$

i.e. A is a subset of $\underline{E}$ such that the value of the constraint membership function is at least equal to the value of the objective membership function.

A good understanding of this result compels to state the three following comments :

(2.3.1.1.) The conditions for the function $\sup _{x \in \underline{A}} \mu_{\boldsymbol{F}}(x)$ to be continuous are little restrictives (Ponsard, 1981 b, 1982 a). Among them, we find on 
the one hand the condition that the (non fuzzy) sets of possible consumption and production be compact, and on the other hand the condition that the fuzzy subsets which describe objectives be strictly convex.

Mathematically, it would be indifferent to lay the strict convexity condition on the constraint rather than on the objective, since they have the same part in the decision making. But for economic motives, it is accurate to lay it on the cbjective. Indeed, in the consumer and producer spatial equilibria theory, it guarantees the continuity property of the fuzzy utility functions. Moreover in the producer equilibrium theory, the assumption of increasing returns does not put a problem since the strict convexity condition is not laid on the technological constraint.

(2.3.1.2.) Generally the solution is not unique. Especially the analysis of the unicity conditions points out that the strong convexity condition must take the place of the strict convexity condition.

(2.3.1.3.) In the particular case where the objective is precise and the constraint alone is fuzzy, then the fuzzy economic calculation can be solved by a different and much simpler method (Ponsard, 1982 b).

\subsubsection{Consumers spatial partial equilibria.}

Consumers maximize their fuzzy utilities with their elastic constraints of budget and taking into account the equilibrium prices which are viewed as parameters.

The $j$ th consumer chooses a consumption $x_{j}$ in a non empty fuzzy subset $x_{j}$ of $\underline{x}_{j}$ such that :

$$
x_{j}=U_{j} \cap C_{j}
$$

where $X_{j}$ describes the demand fuzzy subset of the $j$ th consumer.

At the equilibrium, the consumption $x_{j}$ is such that :

$$
\sup _{x_{j} \in \underline{x}_{j}} \mu_{x}\left(x_{j}\right)=\sup _{x_{j} \in \underline{A}} \mu_{u_{j}}\left(x_{j}\right)
$$

$$
\text { with } \underline{A}=\left\{x_{j} ; x_{j} \in \underline{x}_{j}: \mu_{c_{j}}\left(x_{j}\right) \geqslant \mu_{u_{j}}\left(x_{j}\right)\right\}
$$


As the continuity of the function $\sup _{x_{j} \in \underline{A}} \mu_{U_{j}}\left(x_{j}\right)$ implies that $U_{j}$ be strictly convex, a result is that, $\forall x_{j} \in \underline{A}, \sup _{j} \in \underline{x}_{j} \mu_{x_{j}}\left(x_{j}\right)$ is strictly quasi-concave and $x_{j}$ is strictly convex. Therefore $\underline{x}_{j}$ must also be strictly convex.

In the goods supply space, the places where the several commodities are demanded and the respective quantities purchased are obtained since $x_{j}=\left[x_{i j k}\right]$ where $k$ is the index of supply places of the goods indexed by $i$ which are elements of the vector $x_{j}$.

Now let $x=\sum_{j=1}^{m} x_{j}$ be the total consumption and $\underline{x}=\sum_{j=1}^{m} \underline{x}_{j}$ be the total consumption set, to which a fuzzy subset $X, X \subset \underline{X}$, is associated. It is defined as follows.

The total consumption is such that :

$$
\frac{\mu_{x}(x)}{x}=\frac{\hat{x}_{j}\left[x_{j} \sup _{j} \underline{x}_{j}{ }_{x_{j}}\left(x_{j}\right)\right]}{\sum_{j=1}^{m} x_{j}}
$$

This expression is not a quotient. It means that the sum of individual consumptions (under the lines) is equipped with a membership function, denoted by $\mu_{x}$, (above the lines) such that :

$$
\begin{aligned}
\mu_{x}: \underline{x} & \longmapsto[0,1] \\
x & \longmapsto \mu_{x}(x)=\hat{x}_{j}\left[\sup _{j} \in \underline{x}_{j} \mu_{x_{j}}\left(x_{j}\right)\right]
\end{aligned}
$$

We remark that $\sup _{x_{j} \in \underline{x}_{j}} \mu_{x_{j}}\left(x_{j}\right)$ is necessarily non null, $\forall j \in[1, m]$, since any consumer demands at least a consumption which is equal to the 
physiological minimum.

The fuzzy subset $x$ of $\underline{x}$ is such that the membership function of $x$ is equal to the minimum of the membership functions of the individual demands $x_{j}$ to $x_{j}$. Thus we have :

$$
x=\left\{x, \mu_{x} ; \forall x \in \underline{x}: \mu_{x}(x)=\hat{\Lambda}_{j}\left[\sup _{x_{j}} \in \underline{x}_{j} \mu_{x_{j}}\left(x_{j}\right)\right]\right\}
$$

\subsubsection{Producers spatial partial equilibria.}

Producers maximize their fuzzy utilities of profit with their elastic technological constraints and taking into account the equilibrium prices which are viewed as parameters.

The $k$ th producer chooses a production $y_{k}$ in a non empty fuzzy subset $Y_{k}$ of $\underline{Y}_{k}$ such that :

$$
Y_{k}=G_{k} \cap H_{k}
$$

where $Y_{k}$ describes the supply fuzzy subset of the $k$ th producer.

At the equilibrium, the production $y_{k}$ is such that :

$$
\sup _{y_{k} \in \underline{Y}_{-k}} \mu_{Y_{k}}\left(y_{k}\right)=\sup _{y_{k} \in \underline{A}} \mu_{G}\left(y_{k}\right)
$$

with $\underline{A}=\left\{y_{k} ; y_{k} \in \underline{Y}_{k}: \mu_{H_{k}}\left(y_{k}\right) \geqslant \mu_{G_{k}}\left(y_{k}\right)\right\}$

As the continuity of the function $\sup _{y_{k} \in \underline{A}} \mu_{G}\left(y_{k}\right)$ implies that $G_{k}$ be strictly convex, a result is that, $\forall y_{k} \in \underline{A}, \sup _{y_{k}} \in \underline{Y}_{-k} \mu_{Y_{k}}\left(y_{k}\right)$ is strictly quasi-concave and $Y_{k}$ is strictly convex. Therefore, $\underline{-}_{k}$ must also be strictly convex.

In the goods demand space, the places where the several products are supplied and the respective quantities sold are obtained since $y_{k}=\left[y_{i j k}\right]$ 
where $j$ is the index of demand places of the goods indexed by $i$ which are elements of the vector $y_{k}$.

Now let $y=\sum_{k=1}^{r} y_{k}$ be the total production and $\underline{Y}=\sum_{k=1}^{r} \underline{Y}_{k}$ be the total production set, to which a fuzzy subset $Y, Y \subset \underline{Y}$, is associated. It is defined as follows.

The total production is such that :

$$
\frac{\mu_{Y}(y)}{y}=\frac{\hat{y}_{k}\left[\sup _{k} \in \underline{Y}_{k} \mu_{Y_{k}}\left(y_{k}\right)\right]}{\sum_{\substack{r \\ k=1}} y_{k}}
$$

This expression means that the sum of individual productions (under the lines) is equipped with a membership function, denoted by $\mu_{Y}$, (above the lines), such that :

$$
\begin{aligned}
\mu_{Y}: \underline{Y} & \longmapsto[0,1] \\
y & \longmapsto \mu_{Y}(y)=\hat{y}_{k}\left[\sup _{y_{k}} \in \underline{Y}_{-k} \mu_{Y_{k}}\left(y_{k}\right)\right]
\end{aligned}
$$

We remark that $\sup _{y_{k} \in \underline{Y}_{k}} \mu_{Y_{k}}\left(y_{k}\right)$ is necessarily non null, $\forall k \in[1, r]$, since a producer does not contribute to the total supply in the inaction assumption.

The fuzzy subset $Y$ of $\underline{Y}$ is such that the membership function of $y$ is equal to the minimum of the membership functions of the individual supplies $y_{k}$ to $Y_{k}$.

Thus we have :

$$
Y=\left\{y, \mu_{Y} ; \forall y \in \underline{Y}: \mu_{Y}(y)=\hat{y}_{k}\left[\sup _{y_{k} \in \underline{Y}_{-k}} \mu_{Y_{k}}\left(y_{k}\right)\right]\right\}
$$


2.3.4. Excess demand fuzzy point-to-set mapping and markets equilibrium.

With the previous definitions, the excess demand in a fuzzy economy, denoted by $e$, is equal to :

$e=x-y-w$

It is such that :

$$
\mu_{|X-Y|}(e)=\left|\mu_{X}(x)-\mu_{Y}(x)\right|
$$

As $x \in \underline{X}$ and $y \in \underline{Y}$, the excess demand $x-y-w$ is an element of a set, denoted by $\underline{E}$, with $\underline{E}=\underline{X}-\underline{Y}-\{w\}$.

Then we define an excess demand fuzzy point-to-set mapping e such that :

e : $\underline{P} \longmapsto \underline{E}$

$$
p \longmapsto e(p)=x(p)-y(p)-w
$$

where $x(p)$ is the value of the demand point-to-set mapping for a price $p$, $y(p)$ the value of the supply point-to-set mapping for a price $p$ and $w$ the initial resources.

In order to simplify the notation, the symbol e designates the excess demand and the excess demand fuzzy point-to-set mapping. No ambiguity is possible in the context.

At the equilibrium, we must verify that $\mathrm{e} \leqslant 0$. We must search for the conditions which ought to be fulfilled by $e(p)$ in order to $p$ be such that $e(p) \leqslant 0$ exists.

\section{Conditions of spatial general equilibrium in a fuzzy economy.}

Mathematical tools are stated by Butnariu (1982) who expresses the existence conditions of fixed points for fuzzy mappings. The following statement is slightly different from his. 
3.1. Fuzzy point-to-set mappings and fixed points.

3.1.1. Let $\underline{X}$ be a non empty set and $\underline{L}$ a lattice with at least two elements, the set of all mappings from $\underline{X}$ in $\underline{L}$ is denoted by $\underline{L} \underline{X}$.

A fuzzy subset $A$ of $\underline{X}$ is an element of $\underline{\underline{L}} \underline{X}$ such that :

$A=\left\{x, \mu_{A} ; \forall x \in \underline{X}: \mu_{A}(x) \in \underline{L}\right\}$

The set of all fuzzy subsets of $\underline{x}$ is denoted by $P(\underline{X})$.

3.1.2. A fuzzy point-to-set mapping over a set $\underline{X}$ is a mapping, denoted by $\Gamma$, from $\underline{X}$ to $P(\underline{X})$ which associates an element of $\mathscr{P}(\underline{X})$ to any $x$ of $\underline{X}$ :

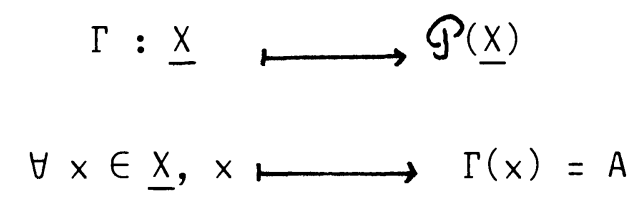

We have :

$\Gamma(x)=\left\{y, \mu_{x} ; y \in \underline{x}, y=f(x): \mu_{x}(y) \in \underline{\underline{L}}\right\}$

Thus, $x$ is a parameter. Giving a particular value to $y$, we can write :

$\Gamma_{x}(y)=\left\{y, \mu_{\Gamma} ; y \in \underline{x}: \mu_{\Gamma}(x, y) \in \underline{L}\right\}$

where $\mu_{\Gamma}$ is the membership function of the couple $(x, y)$ to the fuzzy subset $\Gamma_{x}(y)$.

3.1.3. An element $x^{*}$ of $\underline{x}$ is called fixed point of the fuzzy point-to-set mapping $\Gamma$ iff its membership function to $A$ is at least equal to the membership function of any element $y$ of $\underline{x}$, i.e. iff $\mu_{x^{*}}\left(x^{*}\right) \geqslant \mu_{x^{*}}(y), \forall y \in \underline{x}$. In other words, a fixed point of a fuzzy point-to-set mapping $\Gamma$ over $\underline{x}$ is an element $x^{*}$ such that $\mu_{\Gamma}\left(x^{*}, x^{*}\right) \geqslant \mu_{\Gamma}\left(x^{*}, x\right), \forall x \in \underline{x}$.

This definition is a generalization of the usual one. Indeed, if $\underline{\underline{L}}=\{0,1\}$, we find again the definition of an ordinary point-to-set mapping : 
$y \in f(x) \Leftrightarrow \mu_{x}(y)=1$. Hence, if $\mu_{x^{*}}\left(x^{*}\right) \geqslant \mu_{x^{*}}(y)$, then $\mu_{x^{*}}\left(x^{*}\right)=1$ and $x^{*} \in f\left(x^{*}\right)$.

3.1.4. Let $\underline{C}$ be a subset of $\underline{x}$. The fuzzy point-to-set mapping $\Gamma$ is closed iff the membership function $\mu_{\Gamma}(x, y)$ is upper-semi continuous over $\underline{c}$.

3.1.5. The fuzzy point-to-set $\Gamma$ is convex iff its membership function is quasi-concave.

3.1.6. If $\underline{x}$ is a real topological vector space, locally convex and Hausdorff-separated, $\underline{C}$ a non empty, convex and compact subset of $\underline{x}$, and $\Gamma$ a fuzzy point-to-set mapping over $\underline{c}$, an usual point-to-set mapping, denoted by $\hat{\Gamma}$, can be associated to $\Gamma$ by :

$$
\hat{\Gamma}_{x}(y)=\left\{y ; y \in \underline{c}: \hat{\Gamma}_{x}(y)=\underset{z \in \underline{c}}{V} \Gamma_{x}(z)\right\} .
$$

3.2. Fuzzy function and fixed point theorem.

\subsubsection{Definitions.}

(3.2.1.1.) A fuzzy point-to-set mapping $\Gamma$ over $\underline{c}, \underline{c} \subset \underline{x}$, is said to be a fuzzy function iff, $\forall x \in \underline{c}, \hat{\Gamma}_{x} \neq \emptyset$. It is said to be a "very fuzzy" function iff $\hat{\Gamma}_{x}$ is a mapping over $\underline{\mathrm{C}}$.

(3.2.1.2.) A fuzzy point-to-set mapping over $\underline{\mathrm{C}}$, denoted by $\Gamma^{\prime}$, can be associated with a fuzzy function $\Gamma$, by :

$$
\Gamma_{x}^{\prime}(y)=\left\{\begin{array}{cl}
\Gamma(x, y) & \text { if } y \in \hat{\Gamma}(x) \\
0 & \text { else }
\end{array}\right.
$$

Thus $\hat{\Gamma}^{q}=\hat{\Gamma}$.

Hence it results immediately that $\Gamma$ and $\Gamma^{\prime}$ have the same fixed points and $\Gamma^{\prime}$ is a "very fuzzy" function.

(3.2.1.3.) A fuzzy point-to-set mapping $\Gamma$ over $\underline{c}, \underline{c} \subset \underline{x}$, is said to be F-continuous iff, $\forall x \in \underline{C}$, and for any open set $\underline{D}$ in $\underline{x}$, we have :

$$
\Gamma_{x}(y) \subseteq \underline{D} \cap \underline{C} \Rightarrow \exists \underline{V} \in \underline{V}(y) / \Gamma_{x}(\underline{V}) \subseteq \underline{D} \cap \underline{C}
$$


where $\underline{V}(y)$ is the set of all neighbourhoods of $y$ in $\underline{x}$.

\subsubsection{Fixed point of a fuzzy function.}

Butnariu (1982) generalizes the Brouwer theorem (1909-1910) to the case of a fuzzy function.

(3.2.2.1.) Brouwer theorem. Let $\mathbb{R}^{n}$ be a real topological vector space, locally convex and Hausdorff-separated, $\underline{C}$ be a non empty, convex and compact subset of $\mathbb{R}^{n}$, f a continuous mapping from $\underline{c}$ to $\underline{c}$ such that, $\forall x \in \underline{C}$, the set $\{f(x)\}$ is non empty, convex and compact, then it exists a fixed point $x^{*}$ of $f$, i.e. $\exists x^{*} \in \underline{C} / x^{*}=f\left(x^{*}\right)$.

(3.2.2.2.) Butnariu theorem 1. Let $\underline{C}$ be a non empty, convex and compact subset of $\mathbb{R}^{n}$. If $\Gamma$ is a fuzzy function over $\underline{C}$ and if $\Gamma$ ' is $F$-continuous, then $\Gamma$ has a fixed point in $\underline{C}$.

Proof. The conclusion of paragraph (3.2.1.2.) states that it suffices to prove that $\Gamma^{\prime}$ has a fixed point in $\underline{\mathrm{C}}$.

Let $y=f(x)$ be the unique element of $\underline{c}$ belonging to $\hat{\Gamma}_{x}$. If the function $f: x \longmapsto f(x)$ has a fixed point $x^{*}$ in $\underline{c}$, then $x^{*}=f\left(x^{*}\right) \in \hat{\Gamma}_{x^{*}}$ and the theorem is true.

Since $\underline{C}$ is convex and compact in $\mathbb{R} n$, $f$ has a fixed point in $\underline{c}$ if $f$ is continuous by virtue of the Brouwer theorem. Then it suffices to prove the continuity of $f$ to complete the statement.

Let $x \in \underline{C}$ and $\underline{D}$ be an open set in $\mathbb{R}^{n}$ such that $f(x) \in \underline{D}$.

We have $\Gamma^{\prime}(x) \subseteq \underline{D}$ since $0<\Gamma_{x}^{\prime}(f(x)) \leqslant \underline{D}(x)$ and $y \neq f(x)$ imply that $\Gamma_{x}^{\prime}(y)=0 \leqslant \underline{D}(y)$.

Since $\Gamma^{\prime}$ is $F$-continuous, it exists a neighbourhood $\underline{V} \in \underline{V}(x)$ such that $\Gamma_{x}^{\prime}(\underline{V}) \subseteq \underline{D}$. So $\forall z \in \underline{C}$, we have : $\Gamma_{x}^{q}(\underline{V})(z) \subseteq \underline{D}(z)$.

Let $w \in \underline{V}$. Then :

$0<\Gamma_{w}^{\prime}(f(w)) \leqslant \sup _{v \in \underline{v}} \Gamma_{v}^{\prime}(f(w))=\Gamma_{\underline{\underline{v}}}^{\prime}(f(w)) \leqslant \underline{D}(z)$.

Since $\underline{D}(f(w)) \in\{0,1\}$, we have : $\underline{D}(f(w))=1$ for $w \in \underline{V}$. Thus $f(w) \in \underline{D}, \forall w \in \underline{v}$, i.e. $f(\underline{V}) \subseteq \underline{D}$.

Therefore, we have proved that, $\forall x \in \underline{C}$, with $f(x) \in \underline{D}, \exists \underline{v} \in \underline{V}(x)$ such that $f(\underline{V}) \subseteq \underline{D}$, and $f$ is continuous. 


\subsection{Fuzzy point-to-set mapping and fixed point theorem.}

Butnariu generalizes the Kakutani theorem (1941) to the case of a fuzzy point-to-set mapping.

3.3.1. Kakutani theorem. Let $\mathbb{R}^{\mathrm{n}}$ be a real topological vector space, locally convex and Hausdorff-separated and $\underline{C}$ be a non empty, convex and compact subset of $\mathbb{R}^{n}$. Let $\underline{F}$ be an upper-semicontinuous point-to-set mapping such that : (i) $\forall x \in \underline{C}, \underline{F}(x)$ is a non empty, convex and compact set (ii) the set graph $(\underline{F})=\underset{x \in \underline{C}}{U}(\{x\} \times \underline{F}(x))$ is closed in $\mathbb{R}^{n} \times \mathbb{R}^{n}$, then it exists a fixed point $x^{*}$ of $\underline{F}$ in $\underline{c}$, i.e. $\exists x^{*} \in \underline{c} / x^{*} \in \underline{F}\left(x^{*}\right)$.

\subsubsection{Butnariu theorem 2. Let $\underline{x}$ be a real topological vector space,} locally convex and Hausdorff-separated, and $\underline{C}$ be a non empty, convex and compact subset of $\underline{X}$. If $\Gamma$ is a closed and convex fuzzy point-to-set mapping over $\underline{C}$, then $\Gamma$ has a fixed point in $\underline{C}$.

Proof. The fuzzy point-to-set mapping $\Gamma$ has a fixed point $x^{*}$ iff the point-to-set mapping $\hat{\Gamma}$ has a fixed point, i.e. iff $x^{*} \in \hat{\Gamma}\left(x^{*}\right)$. Indeed $x^{*}$ is a fixed point of $\Gamma$ iff $\mu_{x^{*}}\left(x^{*}\right) \geqslant \mu_{x^{*}}(y)$, which implies that $x^{*} \in \Gamma\left(x^{*}\right)$. Therefore, it suffices to verify that $\hat{\Gamma}$ fulfils Kakutani theorem assumptions.

At first, $\hat{\Gamma}_{x} \neq \emptyset$. Indeed $\hat{\Gamma}_{x}$ is an upper-semicontinuous point-to-set mapping. It has a maximum $y$ and effectively attains it in compact subset $\underline{c}$ of $\mathbb{R}^{n}$. Thus $\exists y \in \underline{c} / y \in \hat{\Gamma}_{x}$ and $\hat{\Gamma}_{x} \neq \emptyset$.

Then, $\hat{\Gamma}_{x}$ is convex. Indeed, let $(y, z)$ be element of $\underline{c}$ and $r \in \underline{L}$, with $\underline{L}=[0,1]$. If $y \in \hat{\Gamma}_{x}$ and $z \in \hat{\Gamma}_{x}$, then $\Gamma_{x}(r y+(1-\bar{r}) z) \geqslant \wedge\left(\hat{\Gamma}_{x}(y), \Gamma_{x}(z)\right)$ by virtue of the quasi-concavity of the membership function of the pointto-set mapping $\Gamma$. Moreover, $\Gamma_{x}(r y+(1-r) z)=\sup _{w \in C} \Gamma_{x}(w)$ by virtue of the definition of $\hat{\Gamma}$. Therefore, $r y+(1-r) z \in \hat{\Gamma}_{x}(z)$ and the property is proved.

Besides, $\forall x \in \underline{C}, \hat{\Gamma}_{x}$ is compact. Indeed, let $\left(x_{i}\right), i \in \underline{I}$, a series in $\hat{\Gamma}_{x}$ whose limit point is $x^{*}$. Then $\Gamma_{x}\left(x_{i}\right) \geqslant \Gamma_{x}(y), \forall y \in \underline{x}$, and $\forall i \in \underline{I}$. From that, $\Gamma_{x}\left(x^{*}\right)=\lim _{i \in \underline{I}} \sup \Gamma_{x}\left(x_{i}\right) \geqslant \Gamma_{x}(y), \forall y \in \underline{c}$, by virtue of the upper-semicontinuous property of $\Gamma_{x}$. Consequently, $\hat{\Gamma}_{x}$ is a closed subset of the compact set $\underline{C}$. So $\widehat{\Gamma}_{x}$ is compact. 
At last, the set graph $\bigcup_{x \in \underline{C}}(\{x\} \times \hat{\Gamma}(x))$ is closed. Indeed, let $\left(x_{i}\right)$ and $\left(y_{i}\right), i \in \underline{I}$, be two series in $\underline{C}$ such that $\left(x_{i}, y_{i}\right)$ be elements of the set graph, $\forall i \in \underline{I}$. Since $\underline{C}$ is compact, $\lim \left(x_{i}\right) \in \underline{C}$. Moreover, $\Gamma_{x_{i}}\left(y_{i}\right) \geqslant \Gamma_{x_{i}}(y), \forall y \in \underline{C}, \forall i \in \underline{I}$.

If $x_{i} \longrightarrow x^{*}$ and $y_{i} \longrightarrow y^{*}$ in $\underline{x}$, therefore in $\underline{c}$ since $\underline{c}$ is compact, then :

$$
\hat{\Gamma}_{x^{*}}\left(y^{*}\right)=\lim _{i \in \underline{I}} \sup \Gamma_{x_{i}}\left(y_{i}\right) \geqslant \lim _{i \in \underline{I}} \sup \Gamma_{x_{i}}(y), \forall y \in \underline{C} .
$$

Since $\Gamma$ is upper-semicontinuous, we have : $\Gamma_{x^{*}}\left(x^{*}\right) \geqslant \Gamma_{x^{*}}(y), \forall y \in \underline{c}$, i.e. $\left(x^{*}, y^{*}\right)$ is an element of the graph and, by definition, the graph is closed.

\subsection{Walras generalized Law.}

3.4.1. Let an excess demand fuzzy point-to-set mapping e which associates the subset $e(p)$ of $\underline{E}$ with any price vector $p$ of $\underline{P}$, such that : $e(p)=x(p)-y(p)-w$.

The Walras Law (1874-1877) can be generalized to the case of a fuzzy spatial economy of competition. It claims that, for all the goods $i$, the sum of the excess demand values is null or negative,

i.e. : $\sum_{i=1}^{n} p \cdot e_{i}(p) \leqslant 0, \forall p \in \underline{P}, \forall i \in[1, n]$.

This condition means that excess demands ought to be non-positive and the goods whose excess demand is negative ought to have a null price. Consequently, according to the definition of a spatial general equilibrium in a fuzzy economy (paragraph 2.3.4.), a competitive equilibrium will be reached if it exists a price vector $p^{*} \in \underline{P}$ and an excess demand vector $e^{*} \in e\left(p^{*}\right)$ such that $e^{*} \leqslant 0$.

3.4.2. If the Walras generalized Law is not verified, a groping process modifies the price levels. In all the markets of goods $i$ such that 
$e_{i}(p) \neq 0$, prices $p_{i j k}$ are rectified by a factor which depends on the excess demand, denoted by $\varepsilon$. Prices remain constant in other cases.

Thus, at any step of the groping process, prices $p^{\prime}{ }_{i j k}$ are substituted to prices $p_{i j k}$ so as :

$$
\begin{aligned}
& p_{i j k}^{\prime}=p_{i j k} \quad \text { if } \quad e_{i}(p)=0 \\
& p^{\prime}{ }_{i j k}=p_{i j k}+\varepsilon \text { if } e_{i}(p)>0 \\
& p^{\prime}{ }_{i j k}=\max \left(0, p_{i j k}-\varepsilon\right) \text { if } e_{i}(p)<0
\end{aligned}
$$

We have a series of continuous functions, denoted by $g$, such that :

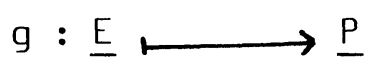

e $\longmapsto g(e)=\left\{p^{\prime} / p^{\prime} . e<p . e\right\}$

In order to verify subsequently the conditions of the fixed points theorems, prices are made standard, i.e. they are such that $\sum_{i, j, k} p_{i j k}=1$.

Let $\underline{\bar{P}}=\left\{\bar{p} ; p \in \underline{P}: \sum_{i, j, k} p_{i j k}=1\right\}$ be the set of standard prices.

The set $\underline{\underline{P}}$ is a simplex.

Thus, a continuous function, denoted $\bar{g}$, is associated with any function $\mathrm{g}$. It is such that :

$$
\begin{aligned}
\bar{g}: & \underline{E} \longmapsto \\
& \text { e } \longmapsto \\
& \bar{g}(e)=\left\{\bar{p}^{\prime} / \bar{p}^{\prime} . e<\bar{p} . e\right\}
\end{aligned}
$$

This transformation brings no loss of generality. The prices of goods in the balanced or excess supply markets are relatively brought down as for the prices of goods in the excess demand markets.

In the same manner, we denote by $\overline{\mathrm{e}}$ the $\mathrm{F}$-continuous fuzzy point-toset mapping, associated with the excess demand fuzzy point-to-set mapping e, which has $\underline{\bar{P}}$ as domain and $\underline{E}$ as co-domain. 
The following theorem states the conditions for which the groping process tends to a price equilibrium in all the markets.

3.4.3. Theorem. The Walras generalized Law is verified if the fuzzy function, denoted by $h$, from the price simplex $\underline{\bar{P}}$ in $\underline{\underline{P}}$ is continuous and if the associated function $h^{\prime}$ is F-continuous.

Proof. Let the fuzzy function $h$ such that :

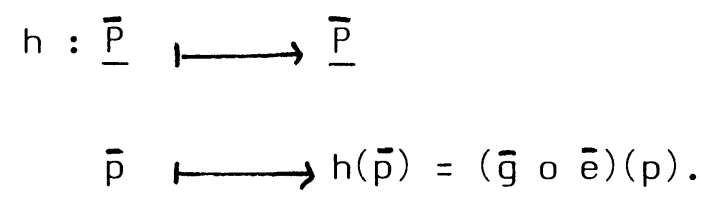

If the fuzzy function $h$ is continuous and if the associated function $h$ ' is $\mathrm{F}$-continuous (see 3.2.1.3.), then the assumptions of the Butnariu theorem 1 are fulfilled since the simplex $\bar{P}$ is a non empty, convex and compact set. Therefore, it exists a fixed point $\bar{p}^{*} \in \underline{\bar{P}}$ which is constant in the transformation $\bar{p} \longmapsto e(\bar{p}) \longmapsto \bar{p}^{\prime}$.

\subsection{Existence of a spatial general equilibrium in a fuzzy economy.}

Now we are ready to express a theorem which states the existence conditions of a spatial competitive equilibrium in a fuzzy economy. It is a generalization of a Debreu theorem (1959) to the case of a spatial economy which is characterized by fuzzy behaviors of agents.

Theorem. Let $\mathbb{R}^{l}$ be a real topological vector space, locally convex and Hausdorff-separated, and $\underline{E}$ be a subset of $\mathbb{R}^{l}$. If the excess demand fuzzy point-to-set mapping $e$ in $\underline{E}$ is closed and has for images $e(p)$ which are non empty and convex, and verifies the Walras generalized Law, then a competitive equilibrium exists in the spatial fuzzy economy, i.e. it exists a price vector $p^{*} \in \underline{P}$ and an excess demand vector $e^{*} \in e\left(p^{*}\right)$ such that $e^{*} \leqslant 0$.

Proof. Let $\underline{E} \subset \mathbb{R}^{1}$. $\underline{E}$ is non empty, convex and compact since, by definition, $\underline{E}=\underline{X}-\underline{Y}-\{w\}$ and the possible consumption and production sets, $\underline{X}$ and $\underline{Y}$ 
respectively, are non empty, compact and strictly convex by virtue of the continuity conditions of the objective functions in the consumers and producers fuzzy economic calculations (see 2.3.1., 2.3.2. and 2.3.3.).

Now let the fuzzy point-to-set mapping, denoted by $\varphi$, such that :

$$
\begin{aligned}
\varphi: & \underline{\bar{P}} \times \underline{E} \longmapsto \underline{\bar{P}} \times \underline{E} \\
& (\bar{p}, e) \longmapsto \varphi(\bar{p}, e)=\{h(\bar{p}), e(\bar{p})\}
\end{aligned}
$$

This fuzzy point-to-set mapping is closed. Indeed, its graph :

$$
G_{\varphi}=\left\{(x, y) \in(\underline{P} \times \underline{E})^{2} / y \in \varphi(x)=G_{h} \times G_{e}\right\}
$$

is closed because it is the product of two closed sets.

Moreover, the fuzzy point-to-set mapping $\varphi$ is convex in $\underline{E}$ since it is the cartesian product of two convex point-to-set mappings. Therefore, the assumptions of Butnariu theorem 2 are verified and the fuzzy point-to-set mapping $\varphi$ has a fixed point in $\underline{E}$.

By virtue of the Walras generalized Law, the fixed point corresponds to a competitive equilibrium state. Indeed, it exists a vector $\left(\bar{p}^{*}, e^{*}\right)$ of $\underline{\underline{P}} \times \underline{E}$ such that :

$$
\left(\bar{p}^{*}, e^{*}\right) \in \varphi\left(\bar{p}^{*}, e^{*}\right)=\left(h\left(\bar{p}^{*}\right), e\left(\bar{p}^{*}\right)\right)
$$

Then we have :

$\left[\bar{p}^{*} \in \mathrm{h}\left(\bar{p}^{*}\right)\right.$ and $\left.e^{*} \in \mathrm{e}\left(\bar{p}^{*}\right)\right] \Rightarrow \forall p \in \underline{p}: p \cdot e^{*} \leqslant p^{*} \cdot e^{*} \leqslant 0$.

Whence $: e^{*} \leqslant 0$. 


\section{Conclusion.}

4.1. The theorem of spatial general equilibrium existence in a fuzzy economy is true whatever be the economic space configuration. Provided that the competitive structure of markets be kept, it is applicable so as to the cases of a space à la Thünen (concentrated demand, scattered supply), a space à la Hotelling (scattered demand, concentrated supply), or a space à la Lösch (scattered demand and supply). At last, it covers, as a particular case, the model of a classical aspatial economy (demand and supply concentrated in a single point).

4.2. However, this result is possible only when space is characterized in a poor manner. It has been described with the help of a set of points which are separated by given distances and all the prices, including the transportation prices, are settled in competitive markets. A richer characterization of space would start notable difficulties. We find again the idea that space is not economically neutral and its integration in the general equilibrium theory put specific problems (Mougeot, 1978).

4.3. The conditions of spatial general equilibrium existence are less constraining in a fuzzy economy than in an ordinary economy. Indeed, the consideration of fuzzy economic calculations permits to state a more general theory and above all to solve some fastidious questions, especially the problem of the equilibrium production with increasing returns. But, the equilibrium conditions are yet restrictive enough, so that a fuzzy economic space, as an usual space, is commonly in a disequilibrium state. On this subject, the theory brings again a conclusion similar to the result of some land-use models (Huriot, 1977). However, the disequilibrium positions in space can be analyzed only by referring to the equilibrium states. For that reason the concern of the spatial general equilibrium theory remains.

4.4. The present paper is incomplete. Several complementary researchs must be looked at. Especially, the stability and optimality properties of the spatial general equilibrium in a fuzzy economy will have to be studied. In the same way, the assumption of competition in markets will 
have to be replaced by that of an oligopolistic economy. The theory wi]l have to be formalized in terms of fuzzy games and fuzzy core. At last, in the present analysis, the locations of the economic agents are given. The difficult problem of the existence of a spatial general equilibrium in a fuzzy economy with endogeneous locations remains open.

\section{Acknowledgements}

The author wishes to thank Mr. M. Prevot (I.M.E.) and the participants to a seminar held at the Catholic University of Louvain, Belgium (March 19, 1984), for fruitful discussions. Of course, he alone is responsible for any shortcomings in the text.

\section{References.}

BROUWER L.E.J. 1909-1910 - On Continuous Vector Distributions on Surfaces. Amsterdam Proceedings, 11-12.

BUTNARIU D. 1982 - Fixed Points for Fuzzy Mappings. Fuzzy Sets and Systems, $7, n^{0} 2,191-207$.

DEBREU G. 1959 - Theory of Value. Cowles Foundation Monograph 17. John Wiley and Sons, Inc. New York.

HURIOT J.M. 1977 - La formation du paysage économique. Essai sur l'affectation de l'espace. Coll. I.M.E., 15, Librairie de l'Université, Dijon.

KAKUTANI S. 1941 - A Generalization of Brouwer's Fixed Point Theorem. Duke Mathematical Journal, 8, 457-459.

MOUGEOT M. 1978 - The Welfare Foundations of Regional Planning : General Equilibrium and Pareto Optimality in a Spatial Economy. Regional Science and Urban Economics, 8, 175-194. 
PIJNSARD C. 1980 - Fuzzy Economic Spaces. First World Regional Science Congress, Harvard University, Cambridge, Massachusetts, June 15-25.

PONSARD C. 1981 a - An Application of Fuzzy Subsets Theory to the Analysis of the Consumer's Spatial Preferences. Fuzzy Sets and Systems, 5, $n^{0} 3$, $235-244$.

PONSARD C. 1981 b - L'équilibre spatial du consommateur dans un contexte imprécis. Sistemi Urbani, 3, 107-133.

PONSARD C. 1982 a - Producer's Spatial Equilibrium with a Fuzzy Constraint. European Journal of Operational Research, 10, $n^{0} 3,302-313$.

PONSARD C. 1982 b - Partial Spatial Equilibria with Fuzzy Constraints. Journal of Regional Science, 22, 159-175.

PONSARD C. 1983 - History of Spatial Economic Theory. Texts and Monographs in Economics and Mathematical Systems. Springer-Verlag, Berlin, Heidelberg, New York, Tokyo.

TANAKA H., OKUDA T. and ASAI K. 1974 - On Fuzzy Mathematical Programming. Journal of Cybernetics, 3, $n^{0} 4,37-46$.

WALRAS L. 1874-1877 - Eléments d'économie politique pure, ou théorie de la richesse sociale. L. Corbaz, Lausanne. 